\title{
On The $Q^{2}$ Dependence of The Spin Structure Function In The Resonance Region
}

\author{
Zhenping $\mathrm{Li}^{1}$ and Zhujun $\mathrm{Li}^{2}$ \\ ${ }^{1}$ Physics Department, Carnegie-Mellon University \\ Pittsburgh, PA. 15213-3890 \\ ${ }^{2}$ CEBAF, 12000 Jefferson Avenue \\ Newport News, VA 23606
}

November 1, 2018

\begin{abstract}
In this paper, we show what we can learn from the CEBAF experiments on spin-structure functions, and the transition from the DrellHearn-Gerasimov sum rule in the real photon limit to the spin dependent sum rules in the deep inelastic scattering, and how the asymmetry $A_{1}\left(x, Q^{2}\right)$ approaches the scaling limit in the resonance region. The spin structure function in the resonance region alone can not determine the spin-dependent sum rule due to the kinematic restriction of the resonance region. The integral $\int_{0}^{1} \frac{A_{1}\left(x, Q^{2}\right) F_{2}\left(x, Q^{2}\right)}{2 x\left(1+R\left(x, Q^{2}\right)\right)} d x$ is estimated from $Q^{2}=0$ to $2.5 \mathrm{GeV}^{2}$. The result shows that there is a region where both contributions from the baryon resonances and the deep inelastic scattering are important, thus provides important information on the
\end{abstract}


high twist effects on the spin dependent sum rule.

PACS numbers: 13.60.Hb, 11.50.Li, 12.40.Aa,14.20Gk 


\section{Introduction}

The polarized lepton nucleon scattering is determined by two spin structure functions $g_{1,2}\left(x, Q^{2}\right)$, which can be written as [1]

$$
g_{1}\left(x, Q^{2}\right)=\frac{M K}{8 \pi^{2} \alpha\left(1+\frac{Q^{2}}{\nu^{2}}\right)}\left[\sigma_{1 / 2}\left(\nu, Q^{2}\right)-\sigma_{3 / 2}\left(\nu, Q^{2}\right)+\frac{2 \sqrt{Q^{2}}}{\nu} \sigma_{T S}\left(\nu, Q^{2}\right)\right]
$$

and

$$
g_{2}\left(x, Q^{2}\right)=\frac{M K}{8 \pi^{2} \alpha\left(1+\frac{Q^{2}}{\nu^{2}}\right)}\left[\frac{2 \nu}{\sqrt{Q^{2}}} \sigma_{T S}\left(\nu, Q^{2}\right)-\left(\sigma_{1 / 2}\left(\nu, Q^{2}\right)-\sigma_{3 / 2}\left(\nu, Q^{2}\right)\right)\right]
$$

where $\sigma_{1 / 2}\left(\nu, Q^{2}\right)$ and $\sigma_{3 / 2}\left(\nu, Q^{2}\right)$ are the cross sections for photon scattering with spin of the photon parallel and antiparallel to the spin of the longitudinallypolarized nucleon with mass $M, \sigma_{T S}\left(\nu, Q^{2}\right)$ represents the interference between the transverse and the longitudinal cross section, and $\mathrm{K}$ is the photon flux. The parton model tells us that the spin dependent structure functions $g_{1,2}\left(x, Q^{2}\right)$ should have simple scaling behaviors in the large $Q^{2}$ limit;

$$
\lim _{Q^{2} \rightarrow \infty} g_{1,2}\left(x, Q^{2}\right) \rightarrow g_{1,2}(x),
$$

and the corresponding spin dependent sum rules [2, 3]

$$
\Gamma=\int_{0}^{1} g_{1}(x) d x
$$

provide us an important information on the spin-flavor correlations of the nucleon. However, the average $Q^{2}$ of the polarized lepton-nucleon scattering experiments may not be high enough to reach the scaling limit, and the $Q^{2}$ dependence of the spin structure function becomes significant. Recent

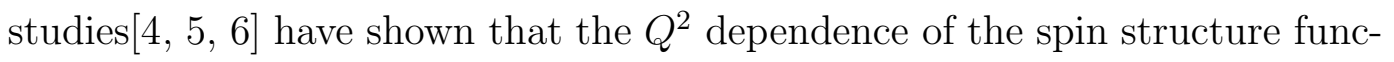
tion should be taken into account in order to explain SMC[7] and SLAC [ $]$ 
measurements on the spin structure function of the nucleon along with the EMC 99] data. Theoretically, such $Q^{2}$ dependence that comes from the high twist effects have been the subject of many studies [10, 11]. The information on the spin structure function in the low $Q^{2}$ region would be more interesting since the high twist effects become stronger, this is where the spin structure function in the resonance region plays an important role. On the other hand, it has been shown 13 that $Q^{2}$ dependence of the spin structure function arises naturally due to the transition from a negative Drell-Hearn-Gerasimov (DHG) sum rule 12] to the positive spin-dependent sum rule in the large $Q^{2}$ limit. The investigations in the explicit quark model show [14] that the DHG sum rule is dominated by the spin structure function in the resonance region, in particular, by the process $\gamma_{v} N \rightarrow P_{33}(1232)$, which has been confirmed by the data 15. How this transition happens in the small $Q^{2}$ region also provides an important test ground for theories, since the explicit quark model [14 and chiral perturbation theory[18] have given different predictions.

The focus of this paper is to examine the $Q^{2}$ dependence of the spin structure function in the resonance region. Our investigation is based on the earlier studies of the $Q^{2}$ extensions of the DHG sum rule[15, [16], and we further replace the photon energy $\nu$ by variable $x$ which is analogue to the Bjorken scaling variable in the deep inelastic scattering. We shall show that the resonance region alone is not sufficient to provide an accurate estimate of the DHG sum rule due to the kinematic restriction. The evolution of the spin asymmetry $A_{1}\left(x, Q^{2}\right)$, which is defined as

$$
A_{1}\left(x, Q^{2}\right)=\frac{\sigma_{1 / 2}\left(x, Q^{2}\right)-\sigma_{3 / 2}\left(x, Q^{2}\right)}{\sigma_{1 / 2}\left(x, Q^{2}\right)+\sigma_{3 / 2}\left(x, Q^{2}\right)},
$$


will also be studied in this framework. A negative DHG sum rule at the real photon limit requires that the spin asymmetry function $A_{1}\left(x, Q^{2}=0\right)$ be negative at the real photon limit. This leads to a transition from a negative $A_{1}\left(x, Q^{2}\right)$ at the real photon limit to the positive $A_{1}\left(x, Q^{2}\right)$ at large $Q^{2}$, the data 20] tell us that such a transition should happen at a relative small $Q^{2}$. If this is indeed to be the case, the $A_{1}\left(x, Q^{2}\right)$ in the resonance region will show how the transition occur. Furthermore, because the DHG sum rule is dominated by the exclusive process $\gamma_{v} N \rightarrow P_{33}(1232)$, one should expect a dramatic $Q^{2}$ dependence in the small $Q^{2}$ region. Thus, there might be a region that both contributions from the resonance region and deep inelastic scattering are important, where the $Q^{2}$ dependence of the structure function is dominated by the leading high twist effects. Our investigation shows that this is possible for $1.0 \leq Q^{2} \leq 2.5 \mathrm{GeV}^{2}$. In the next section, we will establish the connection between the DHG sum rule and the integral of the spin structure function. The $Q^{2}$ evolution of the asymmetry $A_{1}\left(x, Q^{2}\right)$ will also be shown.

Eqs. 11 and 2 show that the DHG sum rule integral $\int_{\nu_{t h}}^{\infty} \frac{\sigma_{1 / 2}\left(\nu, Q^{2}\right)-\sigma_{3 / 2}\left(\nu, Q^{2}\right)}{\nu} d \nu$ used in Refs. [15, 16] is not related to the spin dependent sum rule $\int_{0}^{1} g_{1}\left(x, Q^{2}\right) d x$ at large $Q^{2}$ since the photon flux $K$ in Eqs. 1 and 2 is defined as the equivalent photon energy. Therefore, one should estimate the integral $I\left(Q^{2}\right)=$ $\int_{0}^{1} \frac{A_{1}\left(x, Q^{2}\right) F_{2}\left(x, Q^{2}\right)}{2 x\left(1+R\left(x, Q^{2}\right)\right)} d x$, which is related the DHG sum rule in the real photon limit and the spin dependent sum rules in the deep inelastic scattering region. Furthermore, one has to go beyond the resonance region to give an accurate estimate of the integral $I\left(Q^{2}\right)$. This will be given in section 3. Finally, the conclusion will be given in section 4 . 


\section{The Kinematics}

In order to describe the strong $Q^{2}$ dependence around $Q^{2}=0$ of the spin structure function due to the pion photo and electroproduction that dominate the DHG sum rule, it is more appropriate to define the variable $x$ as

$$
x=\frac{\nu_{t h}}{\nu}
$$

where $\nu_{t h}$ is the threshold energy for the pion-electroproduction, which is related to the pion mass $m_{\pi}$ and the nucleon mass $M$ by

$$
\nu_{t h}=\frac{Q^{2}+2 m_{\pi} M+m_{\pi}^{2}}{2 M} .
$$

The variable $x$ defined in Eq. 6 is exactly the same as variable $\xi$ in Ref. [19], which gives a better description of the strong $Q^{2}$ dependence of the structure function $f_{2}\left(x, Q^{2}\right)$ near threshold. Furthermore, the variable $x$ naturally leads

to the Bjorken scaling variable $x_{B}=\frac{Q^{2}}{2 M \nu}$ in the large $Q^{2}$ limit, and makes it possible to write the DHG integral $\int_{\nu_{t h}}^{\infty} \frac{\sigma_{1 / 2}(\nu)-\sigma_{3 / 2}(\nu)}{\nu} d \nu$ in terms of the integral $\int_{0}^{1} g_{1}\left(x, Q^{2}\right) d x$ at the real photon limit;

$$
\int_{0}^{1} g_{1}\left(x, Q^{2}=0\right) d x=\frac{M \nu_{t h}}{8 \pi^{2} \alpha} \int_{\nu_{t h}}^{\infty} \frac{\sigma_{1 / 2}\left(\nu, Q^{2}=0\right)-\sigma_{3 / 2}\left(\nu, Q^{2}=0\right)}{\nu} d \nu
$$

where the photon flux $K$ in Eq. 1 1 is the photon energy $\nu$ at the real photon limit. The DHG sum rule gives

$$
\int_{0}^{1} g_{1}\left(x, Q^{2}=0\right) d x=-\frac{\nu_{t h}}{4 M} \kappa^{2}=-\frac{2 m_{\pi} M+m_{\pi}^{2}}{8 M^{2}} \kappa^{2}
$$

where $\kappa$ is the anomalous magnetic moments of the nucleon. Substitute $\kappa_{p}=$ 1.79 and $\kappa_{n}=-1.91$ for both protons and neutrons into Eq. 9, we have

$$
\int_{0}^{1} g_{1}\left(x, Q^{2}=0\right) d x=\left\{\begin{array}{lr}
-0.123 & \text { for protons } \\
-0.14 & \text { for neutrons. }
\end{array}\right.
$$


Replacing the photon energy $\nu$ by the variable $x$ at low $Q^{2}$ also shows kinematic restrictions of the resonance region; rewriting the photon energy as

$$
\nu=\frac{W^{2}+Q^{2}-M^{2}}{2 M},
$$

where $W$ is the mass of final state when nucleon $M$ at rest absorbs the incoming photon with energy $\nu$, the resonance region corresponds to the final state mass $W$ from the threshold $W=M+m_{\pi}=1.073$ to $W=1.8 \mathrm{GeV}$ which is also the region that can be reached in the future CEBAF experiments[25]. In Fig. 1 , we show the range of the variable $x$ that is accessible to the resonance region; at $Q^{2}=0$, the resonance region covers $x=0.11 \sim 1$, while $x$ is only limited to $0.57 \sim 1$ at $Q^{2}=2.5 \mathrm{GeV}^{2}$. This raises a serious question whether the resonance region, or the information from the CEBAF experiments, alone would be sufficient to provide an accurate estimate of the spin dependent integral $\int_{0}^{1} g_{1}\left(x, Q^{2}\right) d x$ even at moderate $Q^{2}$.

However, the studies of the spin structure function in the resonance region could provide us very useful information on transition of the spin structure function from the real photon limit to the scaling limit in the deep inelastic scattering. In particular, the data on the spin asymmetry function $A_{1}\left(x, Q^{2}\right)$ in the resonance region will reveal how it reaches the scaling limit, if $A_{1}\left(x, Q^{2}\right)$ has little dependence on $Q^{2}$ after $Q^{2} \geq 0.5 \mathrm{GeV}^{2}$. A significant amount of the pion and $\eta$ electroproduction data has been collected in the resonance region[21], this enable us to extract the transverse photo coupling amplitudes $A_{1 / 2}\left(Q^{2}\right)$ and $A_{3 / 2}\left(Q^{2}\right)$ for the most prominent resonances in the range $0 \leq Q^{2} \leq 3.0$ $\mathrm{GeV}^{2}$. For the other relative weak resonances, we used the single quark transition model to calculate their photo coupling constants. Therefore, combining 
the contribution from the resonances and the background pion production, one can obtain the spin structure function in the resonance region, (for further detail, see [15). In the figure 2 , we show that asymmetry $A_{1}\left(x, Q^{2}\right)$ of proton in the resonance region at $Q^{2}=0,0.5$ and $1.5 \mathrm{GeV}^{2}$, and the data at $Q^{2}=0.5$ and $1.5 \mathrm{GeV}^{2}[20$ are also shown. The asymmetry is mostly negative in the region $x \leq 0.53$ in the real photon limit, since it is dominated by the process $\gamma N \rightarrow P_{33}(1232)$. Moreover, the cancellation between the transitions of spin flip and orbital angular momentum flip for the resonance $F_{15}(1688)$ and $D_{13}(1520)$ [23, 24] also makes helicity amplitudes $A_{1 / 2}$ vanishes for these resonances, which leads to a negative $A_{1}\left(x, Q^{2}=0\right)$ for the higher resonances. As $Q^{2}$ increases, the resonance $P_{33}(1232)$ is shifting to larger $\mathrm{x}$, and the magnetic spin flip transition becomes dominant, thus the asymmetry $A_{1}\left(x, Q^{2}\right)$ becomes positive in the small $\mathrm{x}$ region, and changes the sign and approaches to unity as $x \rightarrow 1$. This tells us that the asymmetry $A_{1}\left(x, Q^{2}\right)$ approaches to the scaling limit in the small $x$ region first, and the range of variable $x$ that asymmetry $A_{1}\left(x, Q^{2}\right)$ scales increases as $Q^{2}$ increases. At the scaling limit, the valence quark model 22] predicts

$$
A_{1}(x)=\frac{19-16 R^{n p}(x)}{15}
$$

where $R^{n p}(x)=\frac{f_{2}^{n}(x)}{f_{2}^{p}(x)}$ is the ratio between the structure functions of neutrons and protons, which give a very good description of the EMC data 9 in the large $x$ region. Comparing with the asymmetry $A_{1}(x)$ in the scaling limit, which is also shown in Fig. 2, we find that the $A_{1}\left(x, Q^{2}\right)$ in the resonance region does not reach the scaling limit at $Q^{2}=0.5 \mathrm{GeV}^{2}$, and it is much closer to the scaling limit at $Q^{2}=1.5 \mathrm{GeV}^{2}$ for $x \leq 0.6$. The future experiments at 
CEBAF 25] would certainly provide the answer in this regard.

\section{The $Q^{2}$ dependence of the spin-dependent sum rule}

There are several problems in estimate the $Q^{2}$ dependence of the integral $\int_{0}^{1} g_{1}\left(x, Q^{2}\right) d x$. First; there is little experimental information available on the longitudinal photon coupling $S_{1 / 2}\left(\nu, Q^{2}\right)$ in the resonance region, we can only estimate the integral

$$
\begin{aligned}
I_{p}\left(Q^{2}\right) & =\int_{0}^{1} \frac{M K}{8 \pi^{2} \alpha\left(1+\frac{Q^{2}}{\nu^{2}}\right)}\left(\sigma_{1 / 2}\left(x, Q^{2}\right)-\sigma_{3 / 2}\left(x, Q^{2}\right)\right) d x \\
& =\int_{0}^{1} \frac{A_{1}\left(x, Q^{2}\right) f_{2}\left(x, Q^{2}\right)}{2 x\left(1+R\left(x, Q^{2}\right)\right)} d x,
\end{aligned}
$$

where $R\left(x, Q^{2}\right)=\sigma_{L}\left(x, Q^{2}\right) / \sigma_{T}\left(x, Q^{2}\right)$ is the ratio between the longitudinal and transverse cross sections, and $K$ is defined as the equivalent photon energy. This integral equals $\int_{0}^{1} g_{1}\left(x, Q^{2}\right) d x$ at the real photon limit and the large $Q^{2}$ limit. Soffer and Teryaev[26] pointed out that the strong $Q^{2}$ dependence of the spin structure function $g_{1}\left(x, Q^{2}\right)$ in the small $Q^{2}$ region might be due to the contamination of the function $g_{2}\left(x, Q^{2}\right)$, this means that the quantity $\sigma_{T S}\left(\nu, Q^{2}\right)$ in Eq. 1 might play an important role in the small $Q^{2}$ region. This might be related to the threshold effect around $Q^{2}=0$, thus one expected it vanish very quickly as $Q^{2}$ increases. The quantitative calculation of $\sigma_{T S}\left(\nu, Q^{2}\right)$ can be done in the framework of valence quark model, and this is in progress. Second, the kinematic restriction shows that the spin structure function in the resonance region alone might not be sufficient to estimate the integral $I_{p}\left(Q^{2}\right)$, Fig. 3 shows the quantity $I\left(x_{\text {min }}\right)=\int_{x_{\text {min }}}^{1} \frac{A_{1}\left(x, Q^{2}\right) f_{2}\left(x, Q^{2}\right)}{2 x\left(1+R\left(x, Q^{2}\right)\right)} d x$ as a function of $x_{\text {min }}$ at $Q^{2}=0$. The DHG sum rule should be extracted at $x_{\text {min }}=0$, and if 
the function $I\left(x_{\min }\right)$ reaches constant in the small $x_{m i n}$ region, it means that the integral $\int_{0}^{x_{\min }} g_{1}\left(x, Q^{2}\right) d x$ can be neglected, thus an accurate DHG sum rule can be obtained experimentally. Unfortunately, Fig. 3 shows that $x_{\text {min }}$ is not small enough to warrant such extraction, and $I\left(x_{\min }\right)$ has not become constant in the small $x_{\min }$ region. Therefore, there is a theoretical uncertainty in the estimate of the DHG sum rule due to the kinematic restrictions of the resonance region, which has not been discussed in the literature [27. The DHG could be obtained by averaging two extreme cases; extrapolating $I\left(x_{\min }=0\right)$ from the function $I\left(x_{\min }\right)$ at small $x_{\text {min }}$ region assuming a smooth behavior of the function $I\left(x_{\min }\right)$ near $x_{\text {min }}=0$, or neglecting the contribution from the outside of the resonance region, and the difference between these two cases represents the theoretical uncertainty. Following this procedure, we find

$$
\int_{0}^{1} g_{1}^{p}\left(x, Q^{2}=0\right) d x=-0.126 \pm 0.011
$$

This result is in good agreement with the theoretical prediction for protons in Eq. 10, and the theoretical uncertainty is quite significant.

Therefore, one can generally write the integral for the spin dependent structure function $I_{p}\left(Q^{2}\right)$ as

$$
I_{p}\left(Q^{2}\right)=I^{r e s}\left(Q^{2}\right)+I^{\text {nonres }}\left(Q^{2}\right)=\int_{x_{r}}^{1}+\int_{0}^{x_{r}} \frac{A_{1}\left(x, Q^{2}\right) f_{2}\left(x, Q^{2}\right)}{2 x\left(1+R\left(x, Q^{2}\right)\right)} d x
$$

where $I^{r e s}\left(Q^{2}\right)$ represents the contribution from the resonance region between $x_{r}$ and 1 , and $I^{\text {nonres }}\left(Q^{2}\right)$ comes from the outside resonance region. The quantity $x_{r}$ in Eq. 15 is

$$
x_{r}=\frac{Q^{2}+2 m_{\pi} M+m_{\pi}^{2}}{W_{r}^{2}+Q^{2}-M^{2}}
$$


with $W_{r}=1.8 \mathrm{GeV}$. Thus, $x_{r}$ approaches to unity as $Q^{2}$ increases, and consequently the contribution from the resonance region decreases. Our result shows that the asymmetry $A_{1}\left(x, Q^{2}\right)$ outside the resonance region may not reach the scaling limit for $x \leq x_{r}$ until $Q^{2} \approx 1 \mathrm{GeV}^{2}$, where the asymmetry data in deep inelastic scattering[9] could be used. Fig. 4 shows our estimate the $Q^{2}$ dependence of the integral $I\left(Q^{2}\right)$. The qualitative behavior of $I^{\text {res }}\left(Q^{2}\right)$ is consistent with the results in Refs. [15, 16], there is a strong $Q^{2}$ dependence of $I^{r e s}\left(Q^{2}\right)$ in the region $0 \leq Q^{2} \leq 0.5 \mathrm{GeV}^{2}$ due to the transition $\gamma_{v} N \rightarrow P_{33}(1232) . I^{r e s}\left(Q^{2}\right)$ becomes positive at $Q^{2} \approx 0.6 \mathrm{GeV}^{2}$ and reaches maximum at $Q^{2}=1.25 \mathrm{GeV}^{2}$. However, $I^{\text {res }}\left(Q^{2}\right)$ is not the whole story, one has to add $I^{\text {nonres }}\left(Q^{2}\right)$ to give an accurate estimate of $I\left(Q^{2}\right)$. Since the asymmetry $A_{1}\left(x, Q^{2}\right)$ in the resonance region shows a strong $Q^{2}$ dependence in $Q^{2} \leq 1 \mathrm{GeV}^{2}$, we only add $I^{\text {nonres }}\left(Q^{2}\right)$ after $Q^{2} \geq 0.75 \mathrm{GeV}^{2}$, in which $A_{1}(x)$ is taken from the EMC data [9], the structure function $f_{2}\left(x, Q^{2}\right)$ is obtained from the parametrization of the experimental data by Donnachie and Landshoff[19], and quantity $R=\sigma_{L}\left(x, Q^{2}\right) / \sigma_{T}\left(x, Q^{2}\right)$ comes from the global fit of the experimental data by Whitlow et al 28]. The result shows that $I^{\text {nonres }}\left(Q^{2}\right)$ gives an dominant contribution, while $I^{\text {res }}\left(Q^{2}\right)$ only accounts for 15 to 20 percent of $I\left(Q^{2}\right)$ in the region $1.0 \leq Q^{2} \leq 2.5 \mathrm{GeV}^{2}$. This is where we believe that one could obtain a reliable information of the high twist effects. If we write the $Q^{2}$ dependence of $I\left(Q^{2}\right)$ above $1 \mathrm{GeV}^{2}$ as

$$
I_{p}\left(Q^{2}\right)=a+\frac{b}{Q^{2}},
$$


our result in $1 \leq Q^{2} \leq 2.5 \mathrm{GeV}^{2}$ region gives

$$
a \approx 0.139 \sim 0.144
$$

and

$$
b \approx-0.03 \sim-0.04 \mathrm{GeV}^{2} .
$$

In particular, the parameter $b$ is consistent with recent analysis of the $\mathrm{SMC}$ and EMC data by Close and Roberts [4] in which they conclude

$$
b=-0.161 \pm 0.530 \mathrm{GeV}^{2}
$$

as well as the QCD sum rule prediction by Balitsky et al[10] used in the analysis by Ellis and Karliner[5].

\section{Conclusion}

We have presented a framework to study the $Q^{2}$ dependence of the spin structure function in the resonance region. At the real photon and large $Q^{2}$ limit, the spin dependent sum rule $I_{p}\left(Q^{2}\right)$ defined in Eq. 13 is related the DHG sum rule and the sum rules in the deep inelastic scattering by

$$
I_{p}\left(Q^{2}\right)=\left\{\begin{array}{cc}
-\frac{\nu_{t h}}{4 M} \kappa^{2} & Q^{2}=0 \\
\Gamma & Q^{2} \rightarrow \infty
\end{array}\right.
$$

which also equals to the integral $\int_{0}^{1} g_{1}\left(x, Q^{2}\right) d x$ in these limits. We show that the data from the resonance region alone is not enough to determine the integral of the spin structure function, even the DHG sum rule in the real photon limit. However, the spin structure function in the resonance region does provides important insights into the transition of the spin structure function as the $Q^{2}$ increases. The DHG sum rule is a threshold effect. It decreases 
very quickly as $Q^{2}$ increases due to its strong connection with the process $\gamma_{v} N \rightarrow P_{33}(1232)$, which is proportional to $Q^{-8}$ at large $Q^{2}$ [14, thus it is not the hight twist effect that generates the leading $1 / Q^{2}$ corrections to the spin structure functions in the deep inelastic scattering region.

Further theoretical and experimental studies of the quantity $\sigma_{T S}\left(\nu, Q^{2}\right)$ is needed, it will not only provide information on the spin structure function $g_{2}\left(x, Q^{2}\right)$ in the small $Q^{2}$ region, but also allow more precise estimation on the $Q^{2}$ dependence of the spin structure function $g_{1}\left(x, Q^{2}\right)$. This investigation is

in progress. Further information on the asymmetry $A_{1}\left(x, Q^{2}\right)$ in the small $x$ region around $Q^{2}=0$ is desirable to give a precise estimate of the DHG sum rule, it may also tell us how the transition from negative to positive $A_{1}\left(x, Q^{2}\right)$ happens quantitatively in the small $x$ region.

We show that there is a region that both contributions from resonance and the nonresonance regions are important, and the effects of the DHG sum rule is small. Therefore, one could obtain the leading high twist effects reliably. Indeed, our result of the $Q^{2}$ dependence of $I\left(Q^{2}\right)$ is quite consistent with the recent theoretical analysis. The future experiments at CEBAF[25] will certainly provide us more information in this area.

Discussions with V. Burkert are gratefully acknowledged. This work is supported by the NSF grant PHY-9023586.

\section{References}

[1] B. L. Ioffe, V.A. Khoze and L. N. Lipatov, Hard Processes, North-Holland, New York (1983). 
[2] J. D. Bjorken, Phys. Rev. 148, 1467(1966); Phys. Rev. D1, 1376(1974).

[3] J. Ellis and R. L. Jaffe, Phys. Rev. D9, 1444(1974); D10, 1669(1974).

[4] F.E. Close and R. G. Roberts, Phys. Lett. B316, 165(1993).

[5] J. Ellis and M. Karliner invited talk at 13th International Conference On Particles and Nuclei, PANIC93, 28 June-2 July 1993, Perugia, Italy, CERN Preprint CERN-TH. 7022/93.

[6] Zhenping Li, Phys. Rev. D48, R3945(1993).

[7] Spin Muon Collaboration, B. Adeva et al., Phys. Lett. 302B, 533(1993).

[8] SLAC-E142 Collaboration, P.L. Anthony et al., Phys. Rev. Lett. 71, 959(1993).

[9] J. Ashman et al, Phys. Lett. 206B, 364(1988); Nucl. Phys. B328, 1(1990).

[10] I.I. Balitsky, V. M. Braun and A. V. Kolesnichenko, Phys. Lett. B242, 245(1990).

[11] X. Ji and P. Unrau, Preprint MIT-CTP-2232, hep-ph/9308263.

[12] S. D. Drell and A. C. Hearn, Phys. Rev. Lett. 162, 1520(1966);

S. B. Gerasimov, Yad. Fiz. 2, 839(1965) [Sov. J. Nucl. Phys. 2, 589(1966)].

[13] M. Anselmino, B. L. Ioffe, and E. Leader, Yad. Fiz. 49, 214(1989)[Sov. J. Nucl. Phys. 49, 136(1989)].

[14] Zhenping Li, Phys. Rev. D47, 1854(1993). 
[15] V. Burkert and Zhujun Li, Phys. Rev. D47, 46(1993).

[16] V. Burkert and B. L. Ioffe, Phys. Lett. 296B, 223(1992).

[17] V.Burkert and Zhujun Li, AO, computer code, unpublised.

[18] V. Bernard, N. Kaiser and Ulf-G. Meissner, Phys. Rev. D48, 3062(1993).

[19] A. Donnachie and P.V. Landshoff, University of Manchester preprint M/C-TH 93/11, DAMTP 93-23, Nucl. Phys. B244, 322(1984).

[20] G. Baum et al, Phys. Rev. Lett. 45, 2000(1980).

[21] F. Foster and G. Hughes, Rep. Prog. Phys. 46, 1445(1983).

[22] F.E. Close, Nucl. Phys. B80, 269(1974); Invited talk at the 6th ICTP Workshop, Trieste, Italy, May 3-7, 1993. RAL preprint RAL-93-034.

[23] F. E. Close and F. Gilman, Phys. Lett. B38, 541(1972).

[24] F. E. Close and Zhenping Li, Phys. Rev. D42, 2194(1990), ibid. D42, $2207(1990)$.

[25] V. Burkert et al, "Measurement of polarized structure functions in inelastic electron proton scattering using the CEBAF large acceptance spectrometer," Report No. CEBAF-PR-91-023(unpublished).

[26] J. Soffer and O. Teryaev, Phys. Rev. Lett. 70, 3373(1993).

[27] I. Karliner, Phys. Rev. D7, 2712(1973); R. Workman and R. Arndt, Phys. Rev. D45, 1789(1992). 
[28] L.W. Whitlow et al Phys. Lett. B250, 193(1990). 


\section{Figure Caption}

1. The shade area is the region of quantity $x$ that can be assessable to the resonance region.

2. The $Q^{2}$ evolution of the spin asymmetry function $A_{1}\left(x, Q^{2}\right)$ in the resonance region; the dot-dashed, short-dashed and solid lines correspond to $A_{1}\left(x, Q^{2}\right)$ [17 at $Q^{2}=0,0.5$ and $1.5 \mathrm{GeV}^{2}$ respectively. The dash-line is the asymmetry $A_{1}(x)$ in the scaling limit 22], and the data are from Ref. [20], which the circle(triangle) represents $A_{1}\left(x, Q^{2}\right)$ at $Q^{2}=0.5(1.5)$ $\mathrm{GeV}^{2}$.

3. The function $I\left(x_{\min }\right)$ as a function of $x_{\text {min }}$ at $Q^{2}=0$. The dashed lines are extensions of $I\left(x_{\min }\right)$ to $I\left(x_{\min }=0\right)$ see text.

4. The $Q^{2}$ dependence of the integral $I^{p}\left(Q^{2}\right)$ the dashed line corresponds to the contribution from the resonance region only, and the solid line is the total result, see text. 
This figure "fig1-1.png" is available in "png" format from: http://arxiv.org/ps/hep-ph/9402308v1 
This figure "fig1-2.png" is available in "png" format from: http://arxiv.org/ps/hep-ph/9402308v1 
This figure "fig1-3.png" is available in "png" format from: http://arxiv.org/ps/hep-ph/9402308v1 
This figure "fig1-4.png" is available in "png" format from: http://arxiv.org/ps/hep-ph/9402308v1 